\title{
Molecular Characterization of Salmonella Enteritidis: Comparison of an Optimized Multi-Locus Variable-Number of Tandem Repeat Analysis (MLVA) and Pulsed-Field Gel Electrophoresis
}

\author{
Isabelle Dewaele, Geertrui Rasschaert, ${ }^{1}$ Sophie Bertrand, ${ }^{2}$ Christa Wildemauwe, ${ }^{2}$ Pierre Wattiau, ${ }^{3}$ \\ Hein Imberechts, ${ }^{3}$ Lieve Herman, ${ }^{1}$ Richard Ducatelle, ${ }^{4}$ Koen De Reu, and Marc Heyndrickx ${ }^{1,4}$
}

\begin{abstract}
Salmonella Enteritidis (SE) is a genetically homogenous serovar, which makes optimal subtype discrimination crucial for epidemiological research. This study describes the development and evaluation of an optimized multiple-locus variable number tandem-repeat assay (MLVA) for characterization of SE. The typeability and discriminatory power of this MLVA was determined on a selected collection of $60 \mathrm{SE}$ isolates and compared with pulsed-field gel electrophoresis (PFGE) using restriction enzymes XbaI, NotI, or SfiI. In addition, the estimated Wallace coefficient $(W)$ was calculated to assess the congruence of the typing methods. Selection of epidemiologically unrelated isolates and more related isolates (originating from layer farms) was also based on the given phage type (PT). When targeting six loci, MLVA generated 16 profiles, while PFGE produced 10, 9, and 16 pulsotypes using XbaI, NotI, and SfiI, respectively, for the entire strain collection. For the epidemiologically unrelated isolates, MLVA had the highest discriminatory power and showed good discrimination between isolates from different layer farms and among isolates from the same layer farm. MLVA performed together with PT showed higher discriminatory power compared to PFGE using one restriction enzyme together with PT. Results showed that combining PT with the optimized MLVA presented here provides a rapid typing tool with good discriminatory power for characterizing $\mathrm{SE}$ isolates of various origins and isolates originating from the same layer farm.
\end{abstract}

\section{Introduction}

Salmonella EnTERitidis (SE) is a major cause of food$\checkmark$ borne illness in humans, in part because of its relation to eggs (EFSA, 2010; Gantois et al., 2009). Epidemiological studies have been performed to study the relatedness of isolates from human infections to contaminated eggs. Typing is a powerful tool to investigate outbreaks and to study the sources and transmission routes in the human and veterinary context (Lapuz et al., 2007; Much et al., 2009). However, availability of large number of genotypic and phenotypic methods (Foley et al., 2007; Kang et al., 2009) complicates selection of the most appropriate technique for characterizing SE. Because SE is one of the most genetically homogenous serotypes of Salmonella (Saeed et al., 2006), methods with high discriminatory power are needed. Traditionally, SE isolates have been characterized by phage typing (PT), a universally applied phenotypic method (De Lappe et al., 2009; Pang et al., 2005). The major advantage of PT is that it is a globally accepted method and specific phage type numbers can be assigned to isolates, which makes comparison between isolates possible on a worldwide scale. In addition, PT has good intralaboratory reproducibility (Majtanova et al., 2011). However, some strains are non-typeable, and possible phage type conversion (Brown et al., 1999; Chart et al., 1989; TankouoSandjong et al., 2012; Threlfall et al., 1989) can occur within the serotype. Some phage types can also predominate in a geographical area, which can limit the utility of PT for investigating local outbreaks (Lukinmaa et al., 1999). Another disadvantage is that only a limited number of reference laboratories perform PT (Cho et al., 2008; Majtanova et al., 2011). Pulsed-field gel electrophoresis (PFGE) using XbaI is another standard method for genotyping SE (Laconcha et al., 2000; Rivoal et al., 2009). The advantages of PFGE are its relatively

\footnotetext{
${ }^{1}$ Technology and Food Science Unit, Institute for Agricultural and Fisheries Research, Melle, Belgium.

${ }^{2}$ National Reference Centre for Salmonella and Shigella, Bacterial Diseases Division, Scientific Institute of Public Health, Brussels, Belgium.

${ }^{3}$ Veterinary and Agrochemical Research Centre, Brussels, Belgium.

${ }^{4}$ Department of Pathology, Bacteriology, and Poultry Diseases, Faculty of Veterinary Medicine, Merelbeke, Ghent University, Belgium.
} 
good discriminatory power and good reproducibility. The PFGE method is labor-intensive and time-consuming (Foxman et al., 2005), which makes it less suitable for typing a large number of isolates. More recently, multilocus variable number of tandem repeat analysis (MLVA) involving amplification and fragment size analysis of the number of repeats in the variable number tandem repeat (VNTR) regions has been documented (Van Belkum, 2007). Good reproducibility, good discriminatory power, and the ease of performance and interpretation make MLVA a valuable technique (Kruy et al., 2011).

The aim of the present study was to compare an optimized MLVA with PFGE for typing SE isolates of various origins and different isolation years as well as typing isolates originating from the same layer farm within the same timeframe. If MLVA has comparable discriminatory power to PFGE, this user-friendly technique could replace the elaborate PFGE method performed together with PT. To make this comparison, we first optimized MLVA using a selection of primers from three existing MLVA systems described in literature. We then selected 60 SE isolates previously characterized by PT and used them to compare the optimized MLVA technique and PFGE using restriction enzymes XbaI, NotI, and SfiI. Typeability and discriminatory power were determined for each method separately, and the Wallace coefficient combining the different methods was calculated.

\section{Materials and Methods}

\section{Development and optimization of MLVA}

Eight characterized SE strains (strains 1-8) of different origins, year of isolation, and/or phage type, and four characterized related outbreak strains (strains 9-12) (Table 1, panel 1) were used to evaluate the typeability and discriminatory power of 25 previously described SE MLVA primer sets (Beranek et al., 2009; Boxrud et al., 2007; Cho et al., 2007, 2008; Malorny et al., 2008; Ross and Heuzenroeder, 2009).

Strains were grown overnight on tryptone soy agar (TSA) plates (Oxoid, Basingstoke, UK) at $37^{\circ} \mathrm{C}$. A small loopful of cells were resuspended in $200 \mu \mathrm{L}$ of high-pressure liquid chromatography (HPLC) water. After incubation during $17 \mathrm{~min}$ at $90^{\circ} \mathrm{C}$, lysates were stored at $-20^{\circ} \mathrm{C}$ until further use. Lysates were centrifuged for $2 \mathrm{~min}$ at $14,000 \times g$ before use in polymerase chain reaction (PCR).

Primers defined by Beranek et al. (2009), Boxrud et al. (2007) Cho et al. (2007, 2008), Malorny et al. (2008), and Ross and Heuzenroeder (2009) were tested separately using the described corresponding PCR protocol to evaluate the typeability and discriminatory power of each primer pair. PCR products were analyzed by electrophoresis in $1.5 \%$ Seakem LE agarose (Lonza, Rockland, ME) with $0.5 \times$ Tris-acetateEDTA (TAE) for $240 \mathrm{~min}$ at $120 \mathrm{~V}$ using a 100-bp DNA size standard (Invitrogen, Carlsbad, CA).

Primer pairs generating none or multiple amplicons for each of the 12 SE isolates of panel 1 were excluded. The final MLVA included six primer pairs. Each pair discriminated among the nine strains of different origin, year, phase type, or all three (as expected, the outbreak isolates showed no difference in band size). Each pair also generated only one specific amplicon. One primer in each pair was labeled with one of the following dyes: PET, 6-FAM, or VIC. This ensured accurate assignment of PCR products to a specific VNTR locus after capillary electrophoresis. Table 2 lists the selected VNTR loci and forward primers with their corresponding fluorescent label.

The optimized MLVA protocol was obtained as follows. Template DNA was prepared as described above. PCR was performed using the Qiagen Type-it Microsatellite PCR Kit (model 206243; Qiagen, Hilden, Germany) in two mixes, each in a total volume of $25 \mu \mathrm{L}$. The first PCR reaction contained $12.5 \mu \mathrm{L}$ of mastermix, $2.5 \mu \mathrm{L}$ of Q-solution, $3.2 \mu \mathrm{M}$ of primer SE7b, $0.04 \mu \mathrm{M}$ of primer SE9, $0.08 \mu \mathrm{M}$ of primer ENTR13, $0.12 \mu \mathrm{M}$ of primer SENTR6, and $1 \mu \mathrm{L}$ of template DNA. The second PCR reaction contained $12.5 \mu \mathrm{L}$ of mastermix, $2.5 \mu \mathrm{L}$ of Q-solution, $0.16 \mu \mathrm{M}$ of primer SE5, $0.12 \mu \mathrm{M}$ of primer SENTR1, and $1 \mu \mathrm{L}$ of template DNA. PCR reactions were performed in a GeneAmp 9700 PCR system (Applied Biosystems, Foster City, CA). Cycling conditions for the first PCR reaction were $94^{\circ} \mathrm{C}$ for $5 \mathrm{~min}$, followed by 30 cycles of $94^{\circ} \mathrm{C}$ for $30 \mathrm{sec}, 62^{\circ} \mathrm{C}$ for $1 \mathrm{~min}$, and $72^{\circ} \mathrm{C}$ for $1 \mathrm{~min}$. A final extension of $72^{\circ} \mathrm{C}$ for $5 \mathrm{~min}$ was employed. Cycling conditions for the second PCR reaction were $94^{\circ} \mathrm{C}$ for $5 \mathrm{~min}$, followed by 20 cycles of $94^{\circ} \mathrm{C}$ for $30 \mathrm{sec}, 60^{\circ} \mathrm{C}$ for $1 \mathrm{~min}$, and $72^{\circ} \mathrm{C}$ for $1 \mathrm{~min}$ with a final extension of $72^{\circ} \mathrm{C}$ for $5 \mathrm{~min}$. Both PCR products were mixed in equal amounts before capillary electrophoresis on ABI PRISM ${ }^{\circledR} 3130$ Genetic Analyzer (Applied Biosystems) with the GENESCAN ${ }^{\mathrm{TM}}-1200$ LIZ $^{\circledR}$ Size Standard. Fragment sizes/repeat numbers were assigned for each locus for analysis with BioNumerics software version 6.5 using the MLVA plugin (Applied Maths, Sint-Martens-Latem, Belgium).

\section{Isolate collection for comparison of MLVA and PFGE}

This study included 60 SE isolates used to compare the typeability and discriminatory power of the optimized MLVA and PFGE. The selection included the ATCC 13076 strain plus 47 isolates of various origins, year of isolation, and phage types considered to be epidemiologically unrelated isolates. In addition, we examined 12 isolates (FODSE) from four layer farms (Farms A, B, C, and H), representing four sets of possibly closely related isolates (Table 1, panel 2). PT of the SE isolates was performed according to the PT scheme of (Ward et al., 1987) at the National Reference Centre for Salmonella (Scientific Institute of Public Health, Brussels, Belgium).

\section{PFGE}

Preparation of agarose plugs, cell lysis, and washing of agarose plugs was performed according to the PulseNet protocol (www.cdc.gov/pulsenet/). Plug slices were digested for $18 \mathrm{~h}$ with $30 \mathrm{U}$ of XbaI, NotI, or SfiI (New England BioLabs, Ipswich, MA) with a digestion temperature of $37^{\circ} \mathrm{C}$ for $\mathrm{XbaI}$ and $\mathrm{NotI}$ and $50^{\circ} \mathrm{C}$ for SfiI. DNA fragments were separated by the CHEF mapper (Bio-Rad, La Jolla, CA) in a $1 \%$ Seakem gold agarose (Lonza, Rockland, MA). The running conditions were $6 \mathrm{~V} / \mathrm{cm}$ at $14^{\circ} \mathrm{C}$ in $0.5 \times$ Tris/borate/EDTA (TBE) buffer for $19 \mathrm{~h}$ with a ramping time from 2.16 to $63.8 \mathrm{~s}$ for the XbaI enzyme, $24 \mathrm{~h}$ with a ramping time from 2 to $10 \mathrm{~s}$ for the NotI enzyme, and $24 \mathrm{~h}$ with a ramping time from 2 to $12 \mathrm{~s}$ for the SfiI enzyme. Gels were stained with ethidium bromide, destained in water, and digitally captured under ultraviolet light. PFGE profiles were clustered with BioNumerics version 6.5 (Applied Maths) using Salmonella Braenderup H9812 digested with XbaI as a normalization reference. Similarities between the fingerprints were calculated using the Dice coefficient (with an optimization of $1 \%$ and a position 
Table 1. Salmonella Enteritidis Isolates Used to Evaluate the Typeability and Discriminatory Power of the Multiple-Locus Variable Number Tandem-Repeat Assay Primers Tested (Panel 1) And Salmonella Enteritidis Isolates

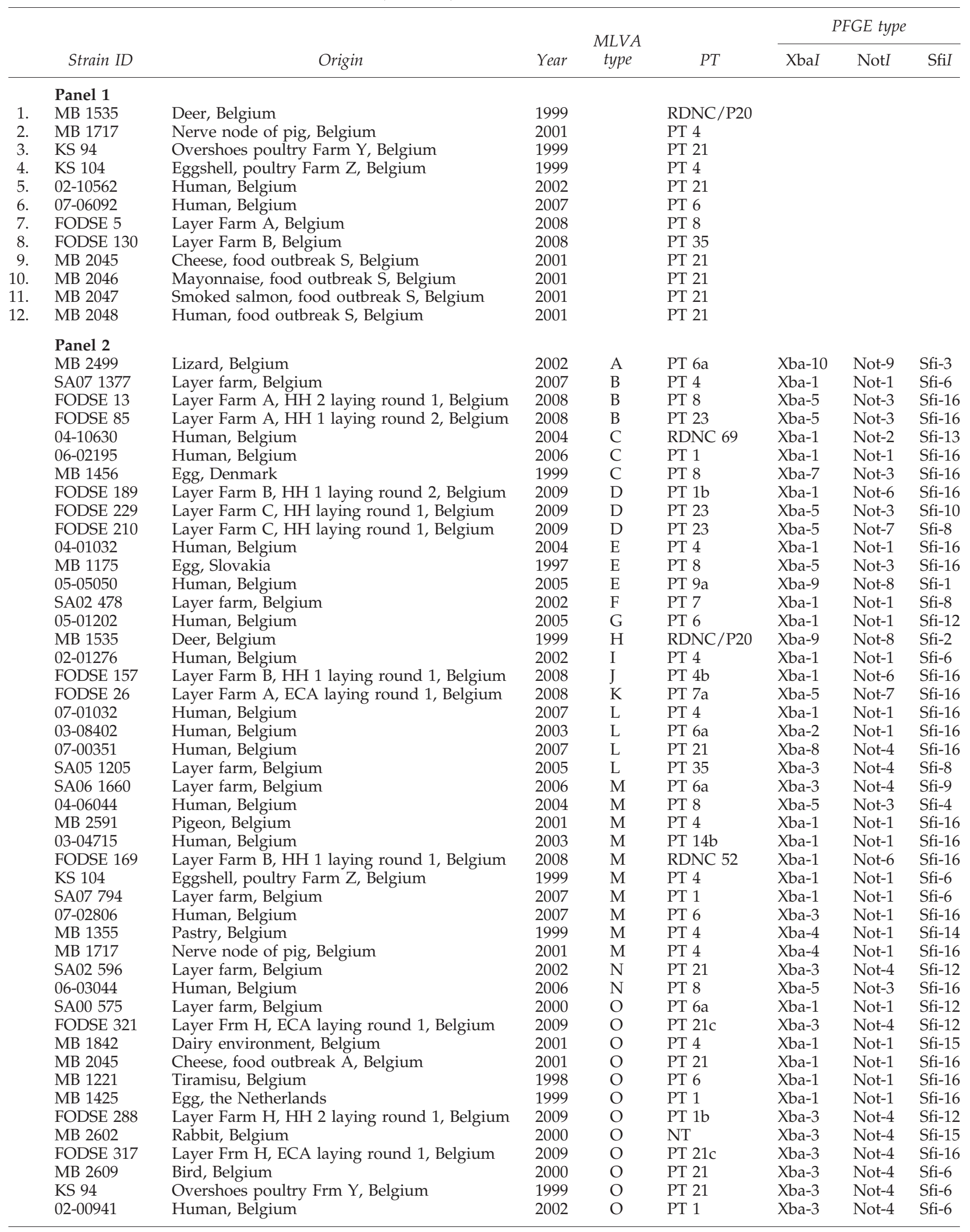


TABle 1. (Continued)

\begin{tabular}{|c|c|c|c|c|c|c|c|}
\hline \multirow[b]{2}{*}{ Strain ID } & \multirow[b]{2}{*}{ Origin } & \multirow[b]{2}{*}{ Year } & \multirow{2}{*}{$\begin{array}{c}M L V A \\
\text { type }\end{array}$} & \multirow[b]{2}{*}{$P T$} & \multicolumn{3}{|c|}{ PFGE type } \\
\hline & & & & & $\mathrm{XbaI}$ & Not $I$ & SfiI \\
\hline FODSE 258 & Layer Frm C, HH laying round 1, Belgium & 2009 & $\mathrm{O}$ & PT 28 & Xba-5 & Not-3 & Sfi-12 \\
\hline 06-02542 & Human, Belgium & 2006 & $\mathrm{O}$ & PT 28 & Xba-5 & Not-3 & Sfi-16 \\
\hline MB 2588 & Sludge, Belgium & 2002 & $\mathrm{O}$ & PT 17 & Xba-1 & Not-1 & Sfi-16 \\
\hline SA00 367 & Layer farm, Belgium & 2000 & $\mathrm{O}$ & PT 14b & Xba-1 & Not-5 & Sfi-16 \\
\hline SA06 407 & Layer farm, Belgium & 2006 & $\mathrm{O}$ & PT 34 & Xba-1 & Not-1 & Sfi-7 \\
\hline SA05 306 & Layer farm, Belgium & 2005 & $\mathrm{O}$ & PT $4 \mathrm{a}$ & Xba-1 & Not-1 & Sfi-8 \\
\hline MB 1418 & Egg, Austria & 1995 & $\mathrm{O}$ & PT 21 & Xba-3 & Not-4 & Sfi-16 \\
\hline $02-09574$ & Human, Belgium & 2002 & $\mathrm{O}$ & PT 21 & Xba-3 & Not-4 & Sfi-16 \\
\hline SA03 1406 & Layer farm, Belgium & 2003 & $\mathrm{O}$ & PT 6 & Xba-3 & Not-4 & Sfi-8 \\
\hline SA03 2252 & Layer farm, Belgium & 2003 & $\mathrm{O}$ & PT 8 & Xba-5 & Not-3 & Sfi-11 \\
\hline 03-08145 & Human, Belgium & 2003 & $\mathrm{O}$ & PT 8 & Xba-6 & Not-3 & Sfi-16 \\
\hline 05-02959 & Human, Belgium & 2005 & $\mathrm{P}$ & PT 34 & Xba-8 & Not-3 & Sfi-5 \\
\hline ATCC $13076^{\mathrm{T}}$ & & & $\mathrm{F}$ & RDNC961 & Xba-5 & Not-3 & Sfi-16 \\
\hline
\end{tabular}

The isolates' corresponding origin, year of isolation, and phage type are noted. Isolates are grouped according to the results obtained by MLVA and PFGE (panel 2).

MLVA, multiple-locus variable number tandem-repeat assay; PFGE, pulsed-field gel electrophoresis; PT, phage typing; RDNC, reacted but did not conform with any standard phage pattern; HH, henhouse; ECA, egg collecting area.

tolerance of $0.7-1.7 \%$ ) and the unweighted-pair group method using arithmetic averages algorithm (UPGMA).

\section{Delineation of MLVA types and pulsotypes}

The VNTR code was defined in the following order: ENTR13 SE5-SE7b-SE9-SENTR1-SENTR6. An MLVA type was assigned based on a difference in repeat numbers of at least one repeat in one VNTR locus. MLVA types were indicated by capital letters. For each PFGE restriction enzyme, a corresponding pulsotype was assigned based on the difference in presence, absence, or clear shift of at least one band in the PFGE fingerprint (Gatto et al., 2006). A pulsotype was indicated by the name of the restriction enzyme followed by a number (e.g., Xba-1).

\section{Calculation of discriminatory power and concordance}

The discriminatory index (DI) was calculated as described by Hunter and Gaston (Hunter, 1990; Hunter and Gaston, 1988) on the collection of 47 epidemiologically unrelated isolates and the ATCC 13076 strain. In addition, Wallace's coefficient $(W)$ was determined together with the proposed Wallace $95 \%$ confidence interval (CI) and Wallace's coefficient under independence (Wi) (Carrico et al., 2006; Pinto et al., 2008). The $W$ coefficient indicates the probability that two isolates classified as the same type by one method will also be classified as the same type when using the other method (Rasschaert et al., 2009). If the $W$ value is not significantly different from the $W i$ value, one can conclude that such congruence of classification could arise by chance.

\section{Results}

\section{Analysis of the 60 SE isolates using MLVA}

All tested SE isolates (Table 1, panel 2) were typeable using the optimized MLVA, except MB 2499, where only two primer pairs (ENTR13, SE9) generated a band. Based on the given VNTR codes, two main clusters and one separate isolate (MB 2499) were generated. In total, 16 allele combinations or MLVA types were found among the 60 SE isolates tested (Table 1 and Fig. 1). VNTR loci SE5 and SENTR6 showed the highest variation.

MLVA was able to discriminate among isolates from different layer farms and between isolates from the same layer farm, except within Farm H. MLVA profiles with their respective pulsotype (XbaI, NotI, and SfiI) and PT type are shown in Figure 1.

Table 2. Primers Selected for Use in the Optimized Multiple-Locus Variable Number Tandem-Repeat Assay

\begin{tabular}{|c|c|c|c|c|}
\hline Locus & Primers & Primer sequence $\left(5^{\prime}-3^{\prime}\right)$ & PCR mix & Reference \\
\hline ush $A$ & $\begin{array}{l}\text { SE9-F } \\
\text { SE9-R }\end{array}$ & $\begin{array}{l}\text { PET-CGTAGCCAATCAGATTCATCCC } \\
\text { GCGTTTGAAACGGGGTGTGGCGCTG }\end{array}$ & 1 & Cho et al., 2007 \\
\hline yohM & $\begin{array}{l}\text { SE5-F } \\
\text { SE5-R }\end{array}$ & $\begin{array}{l}\text { PET-CGGGAAACCACCATCAC } \\
\text { CAGGCCGAACAGCAGGAT }\end{array}$ & 2 & Cho et al., 2007 \\
\hline$y g b F$ & $\begin{array}{l}\text { SE7b-F } \\
\text { SE7b-R }\end{array}$ & $\begin{array}{l}\text { FAM-GATAATGCTGCCGTTGGTAA } \\
\text { ACTGCGTTTGGTTTCTTTTCT }\end{array}$ & 1 & Malorny et al., 2008 \\
\hline Non-coding & $\begin{array}{l}\text { SENTR6-F } \\
\text { SENTR6-R }\end{array}$ & $\begin{array}{l}\text { FAM-ATGGACGGAGGCGATAGAC } \\
\text { AGCTTCACAATTTGCGTATTCG }\end{array}$ & 1 & Malorny et al., 2008 \\
\hline tolA & $\begin{array}{l}\text { SENTR1-F } \\
\text { SENTR1-R }\end{array}$ & $\begin{array}{l}\text { VIC-GCAACAGCAGCAGCAACAG } \\
\text { CCGAGCTGAGATCGCCAAG }\end{array}$ & 2 & Malorny et al., 2008 \\
\hline Non-coding & $\begin{array}{l}\text { ENTR13-F } \\
\text { ENTR13-R }\end{array}$ & $\begin{array}{l}\text { VIC-TATGAACCAATGGCAACGAGAC } \\
\text { CGTGGCAAGGAACAGTAGAGG }\end{array}$ & 1 & Beranek et al., 2009 \\
\hline
\end{tabular}

PCR, polymerase chain reaction. 


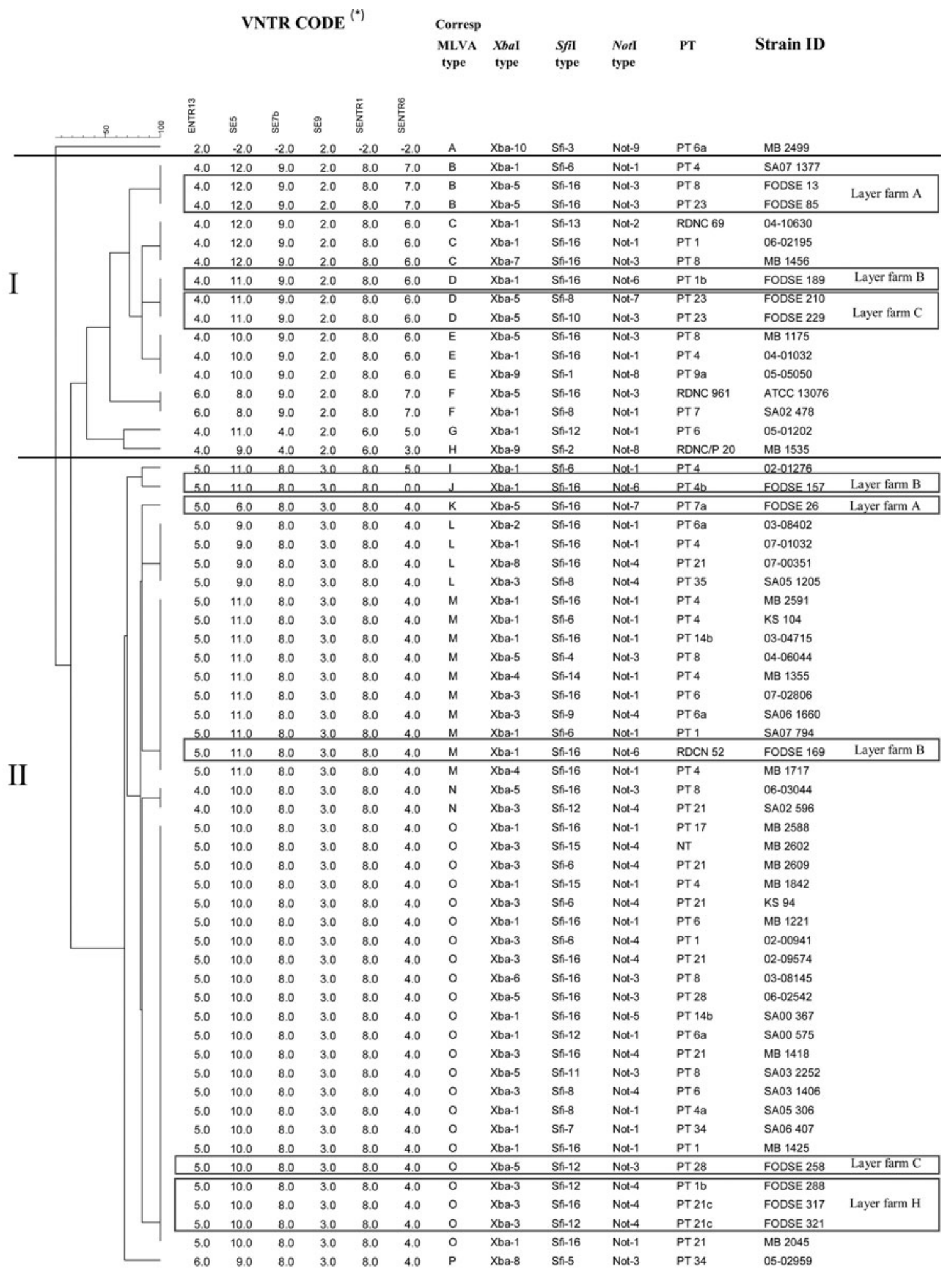

${ }^{(*)}$ VNTR code -2.0 was given (by the Bionumerics software programme) when no feasible band was detected

FIG. 1. Dendrogram and repeat numbers of each variable number tandem repeat (VNTR) locus for multiple-locus variable number tandem-repeat assay (MLVA) performed on 60 Salmonella Enteritidis (SE) isolates. The similarities between the VNTR codes were calculated using categorical values and the fingerprints were grouped according to their similarities using the unweighted-pair group method using arithmetic averages algorithm (UPGMA) algorithm. 


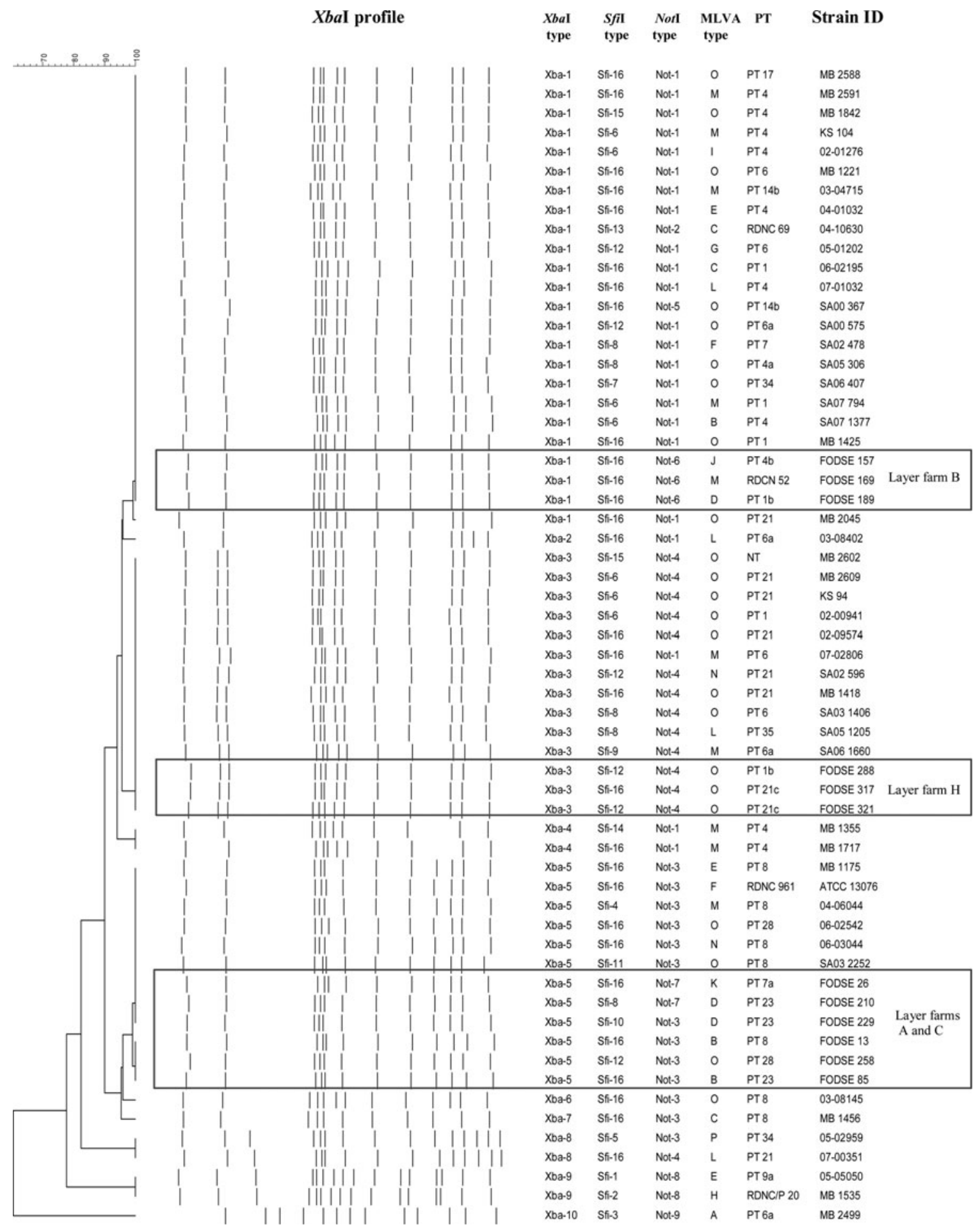

FIG. 2. Dendrogram and fingerprints for pulsed-field gel electrophoresis (PFGE) using restriction enzyme XbaI with 60 Salmonella Enteritidis (SE) isolates. The similarities between the fingerprints were calculated using the Dice coefficient (optimization $1.0 \%$ and position tolerance $1.5 \%$ ), and the fingerprints were grouped according to their Dice similarities using the unweighted-pair group method using arithmetic averages algorithm (UPGMA) algorithm. XbaI pulsotypes are given with their respective NotI and SfiI pulsotype and multiple-locus variable number tandem-repeat assay (MLVA) type.

\section{Analysis of the 60 SE isolates using PFGE}

All isolates (Table 1, panel 2) were typeable by PFGE using XbaI, SfiI, and NotI restriction analysis. A cut-off value of $97 \%$ for $\mathrm{XbaI}$ and SfiI and $96 \%$ for NotI for delineation of the dif- ferent pulsotypes was determined, according to the criteria for the delineation of pulsotypes as described above.

Ten XbaI (Table 1 and Fig. 2) and nine NotI (Table 1 and Fig. 3) pulsotypes were determined within the isolates. Using SfiI (Table 2 and Fig. 4), 16 pulsotypes were distinguished. For 


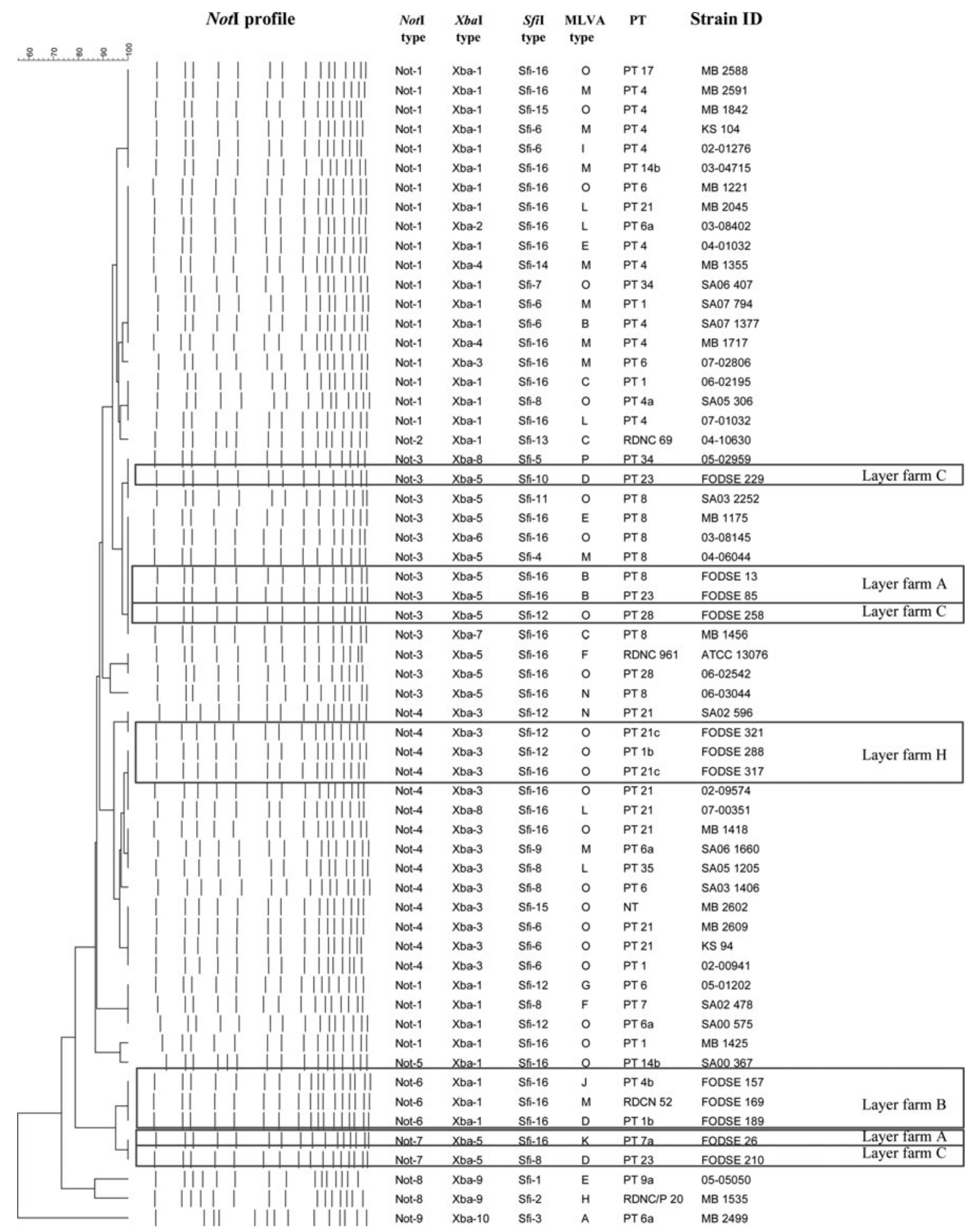

FIG. 3. Dendrogram and fingerprints for pulsed-field gel electrophoresis (PFGE) using restriction enzyme NotI with 60 Salmonella Enteritidis (SE) isolates. The similarities between the fingerprints were calculated using the Dice coefficient (optimization $1.0 \%$ and position tolerance $0.7 \%$ ), and the fingerprints were grouped according to their Dice similarities using the unweighted-pair group method using arithmetic averages algorithm (UPGMA) algorithm. NotI pulsotypes are given with their respective $\mathrm{XbaI}$ and SfiI pulsotype and multiple-locus variable number tandem-repeat assay (MLVA) type.

each method, the MB 2499 isolate formed a separate pulsotype from the other SE isolates.

Using PFGE XbaI or NotI, it was not possible to discriminate between isolates from Farms A and C, whereas PFGE using Sfil found the same pulsotypes on Farms A and B. PFGE using
NotI was restricted to discriminate only within isolates recovered from Farm A or from Farm C. PFGE using SfiI could discriminate within isolates within Farm $\mathrm{C}$ or Farm H. PFGE using $\mathrm{XbaI}$ could not discriminate among any isolates within the same farm. 


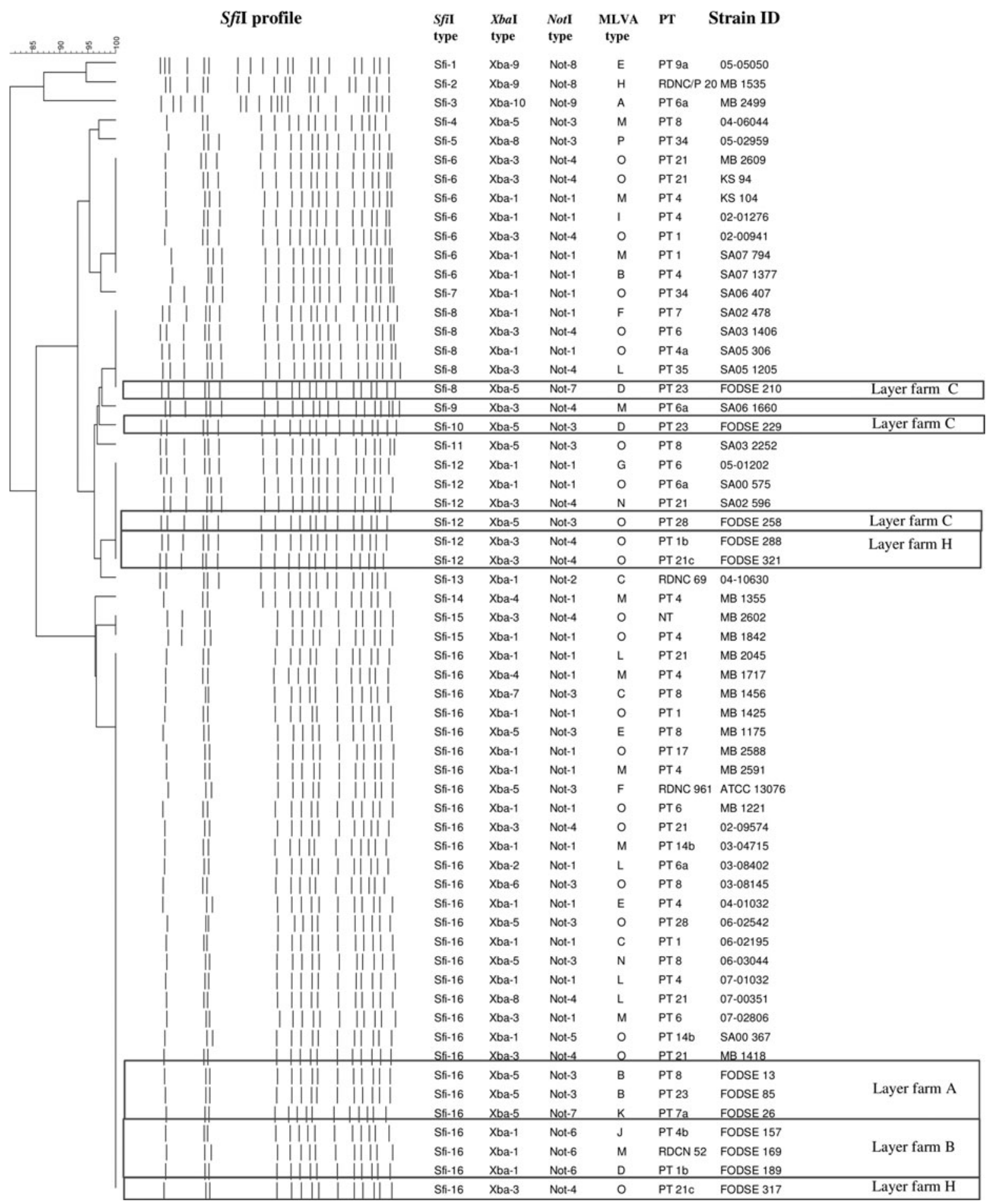

FIG. 4. Dendrogram and fingerprints for pulsed-field gel electrophoresis (PFGE) using restriction enzyme SfiI with 60 Salmonella Enteritidis (SE) isolates. The similarities between the fingerprints were calculated using the Dice coefficient (optimization $1.0 \%$ and position tolerance $1.7 \%$ ), and the fingerprints were grouped according to their Dice similarities using the unweighted-pair group method using arithmetic averages algorithm (UPGMA) algorithm. SfiI pulsotypes are given with their respective $\mathrm{XbaI}$ and NotI pulsotype and multiple-locus variable number tandem-repeat assay (MLVA) type.

\section{Discriminatory power and Wallace coefficient}

The discriminatory index (DI) of each method was determined separately and combined with PT, as calculated for the 48 epidemiologically unrelated SE isolates and the ATCC
13076 strain (layer farm isolates FODSE were not included; Table 3). For each method considered separately, the discriminatory power of PFGE using SfiI, $\mathrm{XbaI}$ or NotI was lower $(\mathrm{DI}=0.77,0.75$, and 0.69 , respectively) compared to MLVA $(\mathrm{DI}=0.80)$. Combining PT with MLVA (DI=0.98) or PFGE 
Table 3. Discriminatory Power of the Various Methods (Individually and in Combination with PT) Evaluated on 48 Epidemiologically UnRelated SE Isolates

\begin{tabular}{|c|c|c|c|c|c|}
\hline Method/combination & No. of types & No. of unique isolates & No. of clustered isolates & Cluster size & $D I$ \\
\hline PFGE NotI & 7 & 3 & 45 & $2-23$ & 0.69 \\
\hline PFGE XbaI & 10 & 4 & 44 & $2-21$ & 0.75 \\
\hline PFGE SfiI & 15 & 10 & 38 & $2-22$ & 0.77 \\
\hline PFGE (all) & 28 & 20 & 28 & $2-9$ & 0.95 \\
\hline MLVA & 13 & 6 & 42 & 2-19 & 0.80 \\
\hline $\mathrm{PT}+\mathrm{PFGE} N o t \mathrm{I}$ & 25 & 19 & 29 & $2-9$ & 0.94 \\
\hline $\mathrm{PT}+\mathrm{PFGE} X b a \mathrm{I}$ & 29 & 21 & 27 & $2-7$ & 0.96 \\
\hline PT + PFGE SfiI & 32 & 23 & 25 & $2-4$ & 0.98 \\
\hline PT + PFGE (all) & 40 & 34 & 14 & $2-3$ & 0.99 \\
\hline $\mathrm{PT}+\mathrm{MLVA}$ & 38 & 33 & 15 & $2-5$ & 0.98 \\
\hline Total & 46 & 44 & 4 & 2 & $1.00^{*}$ \\
\hline
\end{tabular}

*Exact value is 0.998 , because there were two times two clustered isolates, which could not be distinguished using either of the typing methods.

PT, phage typing; SE, Salmonella Enteritidis; DI, discriminatory index; PFGE, pulsed-field gel electrophoresis.

using SfiI $(\mathrm{DI}=0.98)$ resulted in more discriminatory power than combining PT with PFGE using restriction enzyme XbaI or NotI (DI $=0.96$ and 0.94 , respectively).

The congruence between typing methods, expressed by the Wallace coefficient $(W)$, is shown in Table 4 . When comparing PT with another typing method, the highest correlation was found between the information provided by PT and PFGE using NotI in both directions. When comparing MLVA with another typing method, the highest correlation was observed with PFGE using XbaI or NotI. A high bidirectional correspondence between PFGE was seen when using $\mathrm{XbaI}$ and NotI. However, for this data set, $W$ values were very low and most of the calculated 95\% confidence intervals (CI) for $W$ included the respective Wallace coefficient under independence (Wi). This indicates that the congruence of classification could have arisen by chance.

\section{Discussion}

Several MLVA typing schemes for the characterization of SE have been described (Beranek et al., 2009; Boxrud et al., 2007; Cho et al., 2007, 2008; Malorny et al., 2008; Ross and Heuzenroeder, 2009)). However, the use of different loci in each protocol and different primers for the same loci makes it difficult to select the most suitable MLVA scheme. In addition, the different conditions used for running and analysing PCR greatly hinder interlaboratory comparison of the results of the test (Hopkins et al., 2011), which was encountered by our laboratory staff. We therefore evaluated primer pairs from existing MLVA systems for their typeability and discriminatory power and developed an optimized MLVA capillary electrophoresis protocol for the characterization of SE isolates using a new primer combination. Typeability and discriminatory power of this six-locus MLVA were compared with PFGE using restriction enzymes XbaI, NotI, or SfiI on a diverse collection of SE isolates. In this way, we determined the most suitable genotyping method to use in addition to PT. For the different typing methods, we also determined $W$ to analyze correspondence among the classifications of the typing methods. Epidemiologically unrelated (SE isolates with different origins collected over several years) as well as SE isolates sampled on the same layer farm were used to define a suitable subtyping method or a polyphasic approach (combination of typing methods). This enabled us to evaluate their practical use (i.e., a sufficiently high discriminatory power) for the following epidemiological purposes: (i) to distinguish among epidemiologically unrelated SE isolates over several years, (ii) to compare SE isolates originating from layer farms and from human origin, and (iii) to describe contamination routes on SE contaminated layer farms. Results of this study showed that the optimized MLVA method had higher discriminatory power in comparison to PFGE performed with a single restriction enzyme (XbaI, SfiI, or NotI). Only a combination of these three enzymes in PFGE had a considerably higher discriminatory power than MLVA. However, the combination of MLVA with PT had a discriminatory power comparable to combining PT with PFGE using all three enzymes. For any given typing method, $W$ provides an estimate

Table 4. Values of Wi and $W$ with Corresponding 95\% CI for the Typing Methods Between Brackets

\begin{tabular}{lccccc}
\hline & PT & MLVA & PFGE XbaI & PFGE NotI & PFGE SfiI \\
\hline Wi & 0.082 & 0.196 & 0.251 & 0.306 & 0.232 \\
\hline PT & & $0.207(0.055-0.358)$ & $0.478(0.296-0.660)$ & $0.793(0.686-0.901)$ & $0.283(0.141-0.425)$ \\
MLVA & $0.086(0.002-0.169)$ & & $0.303(0.188-0.418)$ & $0.339(0.224-0.455)$ & $0.240(0.094-0.386)$ \\
PFGE XbaI & $0.155(0.056-0.255)$ & $0.237(0.107-0.367)$ & & $0.823(0.650-0.996)$ & $0.233(0.103-0.364)$ \\
PFGE NotI & $0.212(0.097-0.327)$ & $0.217(0.115-0.320)$ & $0.675(0.488-0.863)$ & & $0.278(0.138-0.419)$ \\
PFGE SfiI & $0.099(0.038-0.160)$ & $0.202(0.082-0.323)$ & $0.252(0.111-0.392)$ & $0.366(0.223-0.509)$ & \\
\hline
\end{tabular}

W, Wallace's coefficient; Wi, Wallace's coefficient under independence; PT, phage typing; MLVA, multiple-locus variable number tandemrepeat assay; PFGE, pulsed-field gel electrophoresis. 
of how much new information is obtained from another typing method. Results indicate that partitions defined either by PT, MLVA, or PFGE using XbaI or SfiI could have been best predicted by PFGE using NotI and vice versa. A combination of methodologies likely provides additional information; however, due to the limited number of isolates tested, the estimated $W$ value was very low and in most cases not significantly different from Wi. No reliable information could be obtained on the directional agreement between the typing methods tested (Pinto et al., 2008; Severiano et al., 2011).

All methods showed good discrimination between isolates from different layer farms. However, only the optimized MLVA, PFGE using restriction enzyme SfiI, and PFGE using all three restriction enzymes provided high resolution for SE isolates from the same layer farm.

The optimized MLVA showed good epidemiological concordance because the isolates from a single-strain outbreak were assigned to identical types (data not shown), which was also confirmed by Boxrud et al. (2007). MLVA typing, albeit with a different combination of primers, has been shown to provide enhanced resolution and good reproducibility for characterizing SE (Boxrud et al., 2007; Cho et al., 2007). Cho et al. (2007) also found that MLVA (although with a different combination of primers than ours) had a higher discriminatory power than PFGE combined with PT as tested on various $\mathrm{SE}$ isolates from human and non-human sources. Cho et al. (2010) showed that MLVA (with a composition of seven primers) in combination with PT can be used for effective characterization of SE isolates collected from sporadic human clinical cases. Although they found an association of MLVAbased clusters with phage types using human clinical isolates, this was not confirmed by the present study in which a more diverse and smaller collection of isolates was used.

In conclusion, this optimized MLVA method provides good discriminatory power for characterizing SE isolates. The actual isolate diversity observed by PT could not be obtained by the use of MLVA. A combination of PT and MLVA seems to be providing a higher discriminatory power, as literature and the results obtained from the present study would indicate. In terms of discriminating between SE isolates of different origin and studying contamination routes on a particular layer farm, MLVA, PFGE using SfiI, and PFGE combining all three restriction enzymes can all be used. Nevertheless, MLVA has several advantages over PFGE. MLVA has good discriminatory capacity and has a high throughput because it is a PCR- and capillary-electrophoresisbased technique. These characteristics make MLVA less laborintensive than PFGE, because the data generated are easier to analyze and interpret. MLVA is thus appropriate for epidemiological studies with a large collection of strains.

\section{Acknowledgments}

We thank Rik Lenaerts, Séverine Danschutter, Pieter Siau, and Sabine Van Glabeke for excellent technical assistance, and Miriam Levenson for the English language editing of this manuscript. This research was funded by the Belgian Federal Public Service for Health, Food Chain Safety and Environment (RF 6195).

\section{Disclosure Statement}

No competing financial interests exist.

\section{References}

Beranek A, Mikula C, Rabold P, Arnhold D, Berghold C, Lederer I, Allerberger F, Kornschober C. Multiple-locus variablenumber tandem repeat analysis for subtyping of Salmonella enterica subsp. enterica serovar Enteritidis. Int J Med Microbiol 2009;299:43-51.

Boxrud D, Pederson-Gulrud K, Wotton J, Medus C, Lyszkowicz E, Besser J, Bartkus JM. Comparison of multiple-locus variable-number tandem repeat analysis, pulsed-field gel electrophoresis, and phage typing for subtype analysis of Salmonella enterica serotype Enteritidis. J Clin Microbiol 2007;45:536-543.

Brown DJ, Baggesen DL, Platt DJ, Olsen JE. Phage type conversion in Salmonella enterica serotype Enteritidis caused by the introduction of a resistance plasmid of incompatibility group X (IncX). Epidemiol Infect 1999;122:19-22.

Carrico JA, Silva-Costa C, Melo-Cristino J, Pinto FR, de Lencastre H, Almeida JS, Ramirez M. Illustration of a common framework for relating multiple typing methods by application to macrolide-resistant Streptococcus pyogenes. J Clin Microbiol 2006;44:2524-2532.

Chart H, Row B, Threlfall EJ, Ward LR. Conversion of Salmonella enteritidis phage type 4 to phage type 7 involves loss of lipopolysaccharide with concomitant loss of virulence. FEMS Microbiol Lett 1989;51:37-40.

Cho S, Boxrud DJ, Bartkus JM, Whittam TS, Saeed M. Multiplelocus variable-number tandem repeat analysis of Salmonella Enteritidis isolates from human and non-human sources using a single multiplex PCR. FEMS Microbiol Lett 2007;275:16-23.

Cho S, Whittam TS, Boxrud DJ, Bartkus JM, Saeed AM. Allele distribution and genetic diversity of VNTR loci in Salmonella enterica serotype Enteritidis isolates from different sources. BMC Microbiol 2008;8:146.

Cho S, Whittam TS, Boxrud DJ, Bartkus JM, Rankin SC, Wilkins MJ, Somsel P, Downes FP, Musser KA, Root TP, Warnick LD, Wiedmann M, Saeed AM. Use of multiple-locus variable number tandem repeat analysis and phage typing for subtyping of Salmonella Enteritidis from sporadic human cases in the United States. J Appl Microbiol 2010;108:859-867.

De Lappe N, Doran G, O'Connor J, O'Hare C, Cormican M. Characterization of bacteriophages used in the Salmonella enterica serovar Enteritidis phage-typing scheme. J Med Microbiol 2009;58:86-93.

[EFSA] European Food Safety Authority. Scientific opinion on a quantitative estimation of the public health impact of setting a new target for the reduction of Salmonella in laying hens. EFSA J 2010;8:1546 [86 pp.]. Available at: www.efsa.europa.eu/en/ efsajournal/doc/1546.pdf, accessed August 20, 2012.

Foley SL, Zhao SH, Walker RD. Comparison of molecular typing methods for the differentiation of Salmonella foodborne pathogens. Foodborne Pathog Dis 2007;4:253-276.

Foxman B, Zhang L, Koopman JS, Manning SD, Marrs CF. Choosing an appropiate bacterial typing technique for epidemiologic studies. Epidemiol Perspect Innov 2005;2:10.

Gantois I, Ducatelle R, Pasmans F, Haesebrouck F, Gast R, Humphrey TJ, Van Immerseel F. Mechanisms of egg contamination by Salmonella Enteritidis. FEMS Microbiol Rev 2009;33:718-738.

Gatto AJ, Peters TM, Green J, Fisher IS, Gill ON, O'brien SJ, Maguire C, Berghold C, Lederer I, Gerner-Smidt P, Torpdahl M, Siitonen A, Lukinmaa S, Tschape H, Prager R, Luzzi I, Dionisi AM, van der Zwaluw WK, Heck M, Coia J, Brown D, Usera M, Echeita A, Threlfall EJ. Distribution of molecular subtypes within Salmonella enterica serotype Enteritidis phage type 4 and $S$. Typhimurium definitive phage type 104 in nine 
European countries, 2000-2004: Results of an international multi-centre study. Epidemiol Infect 2006;134:729-736.

Hopkins KL, Peters TM, de Pinna E, Wain J. Standardisation of multilocus variable-number tandem-repeat analysis (MLVA) for subtyping of Salmonella enterica serovar Enteritidis. Euro Surveill 2011;16: pii: 19942.

Hunter PR, Gaston MA. Numerical index of the discriminatory ability of typing systems-An application of Simpsons Index of diversity. J Clin Microbiol 1988;26:2465-2466.

Hunter PR. Reproducibility and indices of discriminatory power of microbial typing methods. J Clin Microbiol 1990;28:19031905.

Kang ZW, Jung JH, Kim SH, Lee BK, Lee DY, Kim YJ, Lee JY, Won HK, Kim EH, Hahn TW. Genotypic and phenotypic diversity of Salmonella Enteritidis isolated from chickens and humans in Korea. J Vet Med Sci 2009;71:1433-1438.

Kruy SL, van Cuyck H, Koeck JL. Multilocus variable number tandem repeat analysis for Salmonella enterica subspecies. Eur J Clin Microbiol Infect Dis 2011;30:465-473.

Laconcha I, Baggesen DL, Rementeria A, Garaizar J. Genotypic characterisation by PFGE of Salmonella enterica serotype Enteritidis phage types $1,4,6$, and 8 isolated from animal and human sources in three European countries. Vet Microbiol 2000;75:155-165.

Lapuz R, Tani H, Sasai K, Shirota K, Katoh H, Baba E. An epidemiological analysis of Salmonella Enteritidis contamination in a rat-infested chicken layer farm, an egg processing facility, and liquid egg samples by pulsed-field gel electrophoresis. J Vet Med Sci 2007;69:649-652.

Lukinmaa S, Schildt R, Rinttila T, Siitonen A. Salmonella Enteritidis phage types 1 and 4: Pheno- and genotypic epidemiology of recent outbreaks in Finland. J Clin Microbiol 1999;37:2176-2182.

Majtanova L, Majtan J, Majtan V. Trends in phage types of Salmonella enterica serovars Enteritidis and Typhimurium isolated in Slovakia from 1995 to 2009. Diagn Microbiol Infect Dis 2011;69:454-456.

Malorny B, Junker E, Helmuth R. Multi-locus variable-number tandem repeat analysis for outbreak studies of Salmonella enterica serotype Enteritidis. BMC Microbiol 2008;8:84.

Much P, Pichler J, Kasper S, Lassnig H, Kornschober C, Buchner A, Konig C, Allerberger F. A foodborne outbreak of Salmonella Enteritidis phage type 6 in Austria, 2008. Wien Klin Wochenschr 2009;121:132-136.

Pang JC, Chiu TH, Chiou CS, Schroeter A, Guerra B, Helmuth R, Tsen HY. Pulsed-field gel electrophoresis, plasmid profiles and phage types for the human isolates of Salmonella enterica serovar Enteritidis obtained over 13 years in Taiwan. J Appl Microbiol 2005;99:1472-1483.

Pinto FR, Melo-Cristino J, Ramirez M. A confidence interval for the Wallace coefficient of concordance and its application to microbial typing methods. PLoS One 2008;3:e3696.

Rasschaert G, Vanderhaeghen W, Dewaele I, Janez N, Huijsdens $\mathrm{X}$, Butaye P, Heyndrickx M. Comparison of fingerprinting methods for typing methicillin-resistant Staphylococcus aureus sequence type 398. J Clin Microbiol 2009;47:3313-3322.

Rivoal K, Protais J, Queguiner S, Boscher E, Chidaine B, Rose V, Gautier M, Baron F, Grosset N, Ermel G, Salvat G. Use of pulsed-field gel electrophoresis to characterize the heterogeneity and clonality of Salmonella serotype Enteritidis, Typhimurium and Infantis isolates obtained from whole liquid eggs. Int J Food Microbiol 2009;129:180-186.

Ross IL, Heuzenroeder MW. A comparison of two PCR-based typing methods with pulsed-field gel electrophoresis in Salmonella enterica serovar Enteritidis. Int J Med Microbiol 2009;299:410-420.

Saeed AM, Walk ST, Arshad M, Whittam TS. Clonal structure and variation in virulence of Salmonella Enteritidis isolated from mice, chickens, and humans. J AOAC Int 2006;89:504511.

Severiano A, Pinto FR, Ramirez M, Carrico JA. Adjusted Wallace coefficient as a measure of congruence between typing methods. J Clin Microbiol 2011;49:3997-4000.

Tankouo-Sandjong B, Kinde H, Wallace I. Development of a sequence typing scheme for differentiation of Salmonella Enteritidis strains. FEMS Microbiol Lett 2012;331:165-175.

Threlfall EJ, Rowe B, Ward LR. Subdivision of Salmonella Enteritidis phage types by plasmid profile typing. Epidemiol Infect 1989;102:459-465.

Van Belkum A. Tracing isolates of bacterial species by multilocus variable number of tandem repeat analysis (MLVA). FEMS Immunol Med Microbiol 2007;49:22-27.

Ward LR, Desa JDH, Rowe B. A phage-typing scheme for Salmonella Enteritidis. Epidemiol Infect 1987;99:291-294.

Address correspondence to: G. Rasschaert, Ph.D.

Technology and Food Science Unit

Institute for Agricultural and Fisheries Research (ILVO)

Brusselsesteenweg 370

9090 Melle, Belgium

E-mail: geertrui.rasschaert@ilvo.vlaanderen.be 